\title{
Tibial rotational osteotomy with intramedullary nail fixation
}

\author{
Alan K. Stotts $\cdot$ Peter M. Stevens
}

Received: 20 February 2009/ Accepted: 12 November 2009/Published online: 26 November 2009

(C) Springer-Verlag 2009

\begin{abstract}
There are several theoretic advantages of using intramedullary rod fixation for tibial osteotomy fixation. We performed a retrospective review of patients who were treated with a mid-diaphyseal osteotomy of the tibia fixed with an intramedullary rod for isolated, symptomatic tibial torsion. Forty patients (59 tibias) were included in the study and were followed for a minimum of 12 months or until rod removal (average follow-up 22.6 months). Major complication rate was $8.5 \%$, which is comparable to alternative methods of fixation. We believe that intramedullary rods are a safe alternative for fixation of tibial rotational osteotomy in patients with physeal closure.
\end{abstract}

Keywords Torsion - Intramedullary rod $\cdot$ Knee pain

\section{Introduction}

Tibial torsion is a well-known phenomenon in children. Most commonly, the torsion spontaneously corrects with age. When it does not, it rarely requires treatment. Symptomatic lateral torsion of the tibia which has failed to correct with growth may be corrected with osteotomy in patients who fail conservative treatment. The excessive lateral twist in the tibia results in excessive valgus force at

A. K. Stotts $(\square)$ · P. M. Stevens

Orthopaedic Surgery, Primary Children's Medical Center,

University of Utah, 100 Mario Capecchi Drive \#4550,

Salt Lake City, UT 84113, USA

e-mail: alan.stotts@hsc.utah.edu the knee $[5,10]$, predisposes the knee to patellar instability $[15,16]$, osteochondral defects [2] and anterior knee pain $[11,16]$.

The osteotomy to correct this torsion and remove the excessive strain on the knee can be fixed by a variety of methods including casting $[1,9,12]$, crossed pins [4, 6, 13] and plates [4, 5, 14]. Casting offers the advantage of no hardware, but potential loss of fixation. Crossed pins that offer the advantage of simple fixation, but require cast protection, are temporary and must be removed early due to potential complications from the pin crossing the skin. Plates offer the advantage of fixation beneath the skin, but the rigid fixation may cause delayed healing of the bone and may not permit early weightbearing. The use of intramedullary rods for fixation of the tibia is attractive since it allows for fixation within the bone-sharing the load of walking with the bone and therefore promoting healing, minimizing soft tissue dissection, avoiding the need for supplemental fixation such as a cast and facilitating early weightbearing. A similar approach of intramedullary fixation has been previously reported for isolated femoral osteotomy in pediatric patients [8].

Ferri-de-Barros et al. [7] described the use of intramedullary nail fixation for tibial rotational osteotomy in 14 patients with cerebral palsy with good results. A group of seven patients with intramedullary rod fixation for tibial rotational osteotomy has been presented by Bruce and Stevens [3]. These patients had no underlying neuromuscular disease but also had contemporaneous femoral osteotomy for pan genu torsion.

The goal of this study was to review the outcome of isolated tibial rotational osteotomy fixed with intramedullary nails in patients with no underlying neuromuscular disease. 


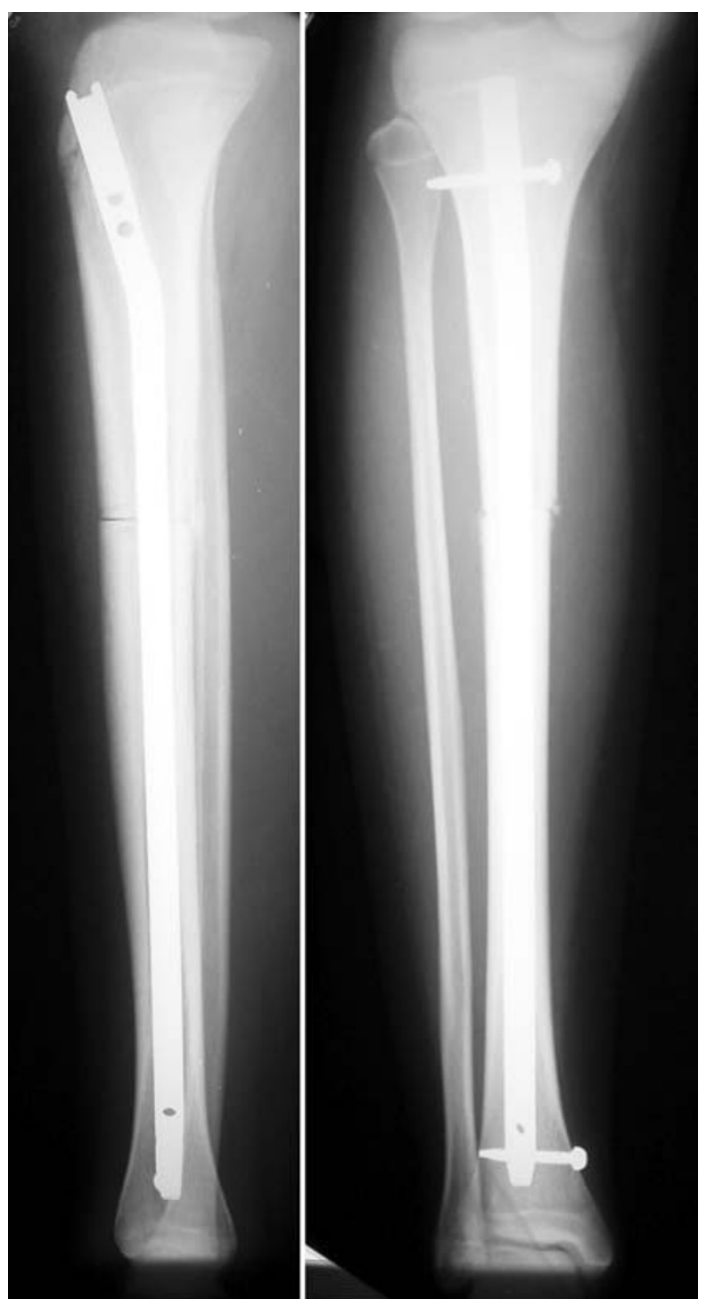

Fig. 1 Two view tibia radiograph demonstrating placement of intramedullary rod across osteotomy 1 month postoperative

\section{Operative technique}

Patients are placed under general anesthetic and epidural catheter is established. Padded tourniquet is placed on the upper thigh and the thigh and leg is prepped and draped. The leg is exsanguinated and the tourniquet inflated. A 4-cm incision is made along the anterior tibial border in the mid-diaphysis and dissection taken to the crural fascia. The fascia is opened and anterior compartment fasciotomy is performed staying close to the lateral margin of the tibia. The tibia is then subperiosteally exposed at this level, and a transverse osteotomy is made through the tibia using a sagittal saw leaving the posterior cortex intact. This facilitates the osteotomy and allows for intramedullary decompression during reaming. A second incision is made at the patellar tendon and dissection carried down to the insertion site for the tibial nail. If a lateral release is to be performed, it is made through this same incision. The tourniquet is then deflated and standard entry is made into

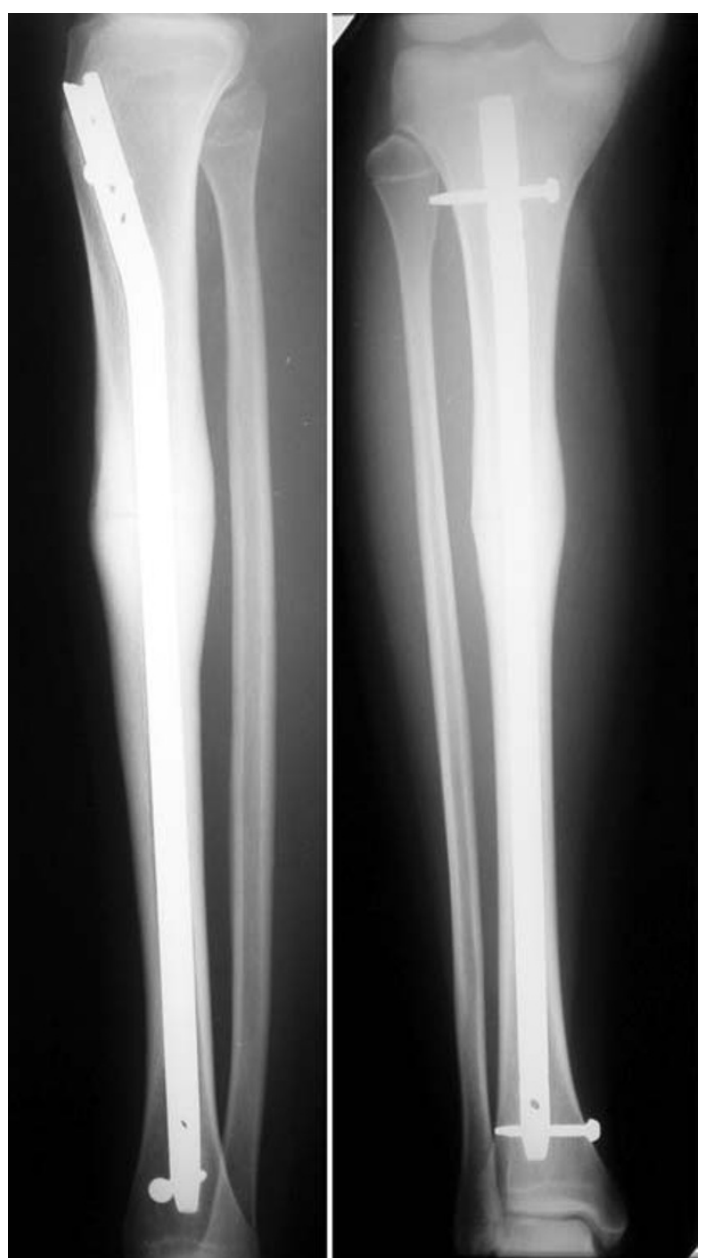

Fig. 2 Two view tibia radiograph showing healing at 8 months

the intramedullary canal, and the tibia is sequentially reamed and rod size determined. A unicortical 5/64" Kirschner wire is then placed on either side of the osteotomy to measure rotation and the osteotomy is completed. The wires are then removed. The rod is placed and locked distally. The wires are replaced in the same holes; the desired rotation is rechecked and measured using the wires and the thigh-foot axis. The rod is then locked proximally using the jig while the rotation is held. We have not found concomitant fibular osteotomy to be helpful $[9,12,13]$. Standard closure is performed, and the leg is wrapped with an Ace bandage. Postoperatively, patients are encouraged to weightbear as tolerated. Figures 1 and 2 demonstrate radiographs of a sample patient in the series 1 and 8 months postoperative.

\section{Materials and methods}

Institutional Review Board approval was obtained for our study. We performed a retrospective review of the charts 
and radiographs of all patients who have had elective rotational osteotomies of the tibia fixed with an intramedullary rod by the senior author (PMS). Charts were reviewed to obtain demographic information, determine symptoms related to the rotation, review operative notes and techniques, identify presence of complications related to the surgery or postoperative period and determine symptoms at final follow-up. Patients were excluded for inadequate chart documentation or lack of follow-up for at least 12 months.

\section{Results}

Forty-six patients were identified as having an isolated elective rotation of the tibia over an intramedullary rod from 1997 to 2004. One patient was excluded for lack of chart notes adequate for review. Two patients had followup elsewhere rendering their charts and films inadequate. Three patients were excluded for follow-up of $<12$ months. Of the remaining 40 patients, 19 had bilateral osteotomies and 21 had unilateral for a total of 59 tibias. Of the bilateral cases, four had the procedures done at the same surgery and the remaining 15 had the procedures staged an average of 11.4 weeks apart (range 5-33 weeks).

There were 36 female patients ( 55 tibias) and four male patients (four tibias). Average age at the time of surgery was 16.5 years (range 12-25 year). All patients had experienced closure of the proximal tibia physis at the time of surgery. All osteotomies were to correct outward tibial torsion.

Fifty-eight of 59 extremities had anterior knee pain with activities preoperatively. One patient did not have complaints of pain but complained of severe torsion. One patient (one tibia) complained of a single prior dislocation of the patella. Five patients (seven tibias) complained of multiple prior episodes of patellar dislocation.

Thirteen of 59 patients $(22 \%$; 19 tibias) had prior procedures related to their rotation or knee symptoms. One patient with juvenile rheumatoid arthritis had multiple steroid injections in each knee. Two patients (four tibias) had previous femoral rotational osteotomies remotely. Nine knees had prior arthroscopy at other facilities-one diagnostic, five included lateral releases, one included plica excision and one had saucerization of a discoid meniscus. Three patients had prior medial transfer of the tibial tubercle. One patient had a previous rotational osteotomy of the ipsilateral tibia treated with a plate. One patient had an open arthrotomy to retrieve a loose osteocartilaginous fragment and perform lateral release.

Thirty-four of 40 (85\%)patients had a preoperative rotation "Gunsight" CT scan of the lower extremities to confirm the amount of torsion. This showed an average of $40.2^{\circ}$ (range $20^{\circ}-67^{\circ}$ ) of outward torsion in the tibia.
Patients who also had more than $20^{\circ}$ of femoral torsion were excluded from this series. All patients had a standing AP radiograph of the legs to ensure a lack of coronal malalignment and lateral radiographs of the knees. Several also had a long standing radiograph taken with the patellae neutral to the cassette and another taken with the feet neutral to demonstrate the torsion.

Forty-nine of $59(83.1 \%)$ extremities had lateral retinacular release performed at the same surgery through the same proximal incision. This was indicated if the lateral patellar retinaculum was tight by attempted passive patellar tilt and medial patellar glide. Of the 10/59 that did not have a lateral release, five of them had had a prior lateral release at a previous surgery. Fifty-seven of $59(96.6 \%)$ extremities had prophylactic fasciotomy of the anterior compartment.

The amount of rotation achieved at the surgery averaged $28.8^{\circ}$ (range $20^{\circ}-50^{\circ}$ ). Fibular osteotomy was performed in the first patient in the series but was not felt to be necessary thereafter and was not used again to achieve the desired rotation. Patients were fixed with an antegrade-locked intramedullary tibial nail.

Three of $59(5.1 \%)$ had an oblique Kirschner wire placed across the osteotomy for additional rotational stability. These patients were early in the series and fixed with a rod that did not permit axial compression. The wire was left out of the skin and pulled in the clinic 4 weeks postoperative. In each of the cases, the wire was used for perceived rotational instability at the time of surgery after the locking of the rod and was used in cases where the rod did not provide compression at the osteotomy site. In one case, the bone was felt to be osteopenic proximally in the tibia, and the proximal locking construct through the rod was considered inadequate to hold the rotation, so a supplemental semitubular plate was placed at the osteotomy site. Later, patients in the series were fixed with a nail that permitted axial compression of the osteotomy.

Epidural catheter was used for postoperative pain control in 53/59 (89.8\%) of cases. There were no compartment syndromes in the series.

Fifty-two of $60(85.2 \%)$ rods were removed at an average of 12.2 months after insertion (range 5.5-24.5 months). All patients were followed for at least 12 months or until removal of the rod. Average follow-up was 22.6 months $(6.75-58.5 \mathrm{~m})$. At final follow-up, $45 / 59(76.3 \%)$ of patients had no pain in the extremity. There was no clinical loss of the rotation achieved intraoperatively in any patient.

Ten of 59 (16.9\%) extremities had at least one complication related to the surgery. Major complications were present in $5 / 59$ patients $(8.5 \%)$. They included peroneal nerve palsy, infection, nonunion and refracture of the osteotomy.

One patient had bilateral staged inward rotations of $40^{\circ}$, and EHL weakness was noted after the first side was done. 
When the second side was done 5 weeks later, she had complete loss of peroneal function and had a peroneal decompression performed on postoperative day number seven. On both sides, the motor returned fully to clinical examination but superficial peroneal numbness remains on the side that had complete initial loss. Her initial rotational profile on CT scan showed that she was $16^{\circ}$ and $14^{\circ}$ retroverted in the femurs and was outward in the tibiae $59^{\circ}$ and $61^{\circ}$. She had no significant mechanical axis deviation. Perhaps, her fully outward profile in the femurs and tibiae permitted less tolerance for her inward rotations.

One patient presented at 1 month with increased pain at the osteotomy site. She had a noncompressing rod at the index surgery and was one of the patients in whom a supplemental Kirschner wire was used. Clinical concern was that she may have increased motion at the osteotomy, so blocking screws were added to the construct. The area was aspirated and cultured. The culture showed Klebsiella oxytoca, and a PICC line was started, and IV antibiotics were given for 4 weeks. The infection resolved without sequelae, and the osteotomy progressed to union.

One patient had removal of her rod 11 months after osteotomy with apparent healing on radiographs. Painless, progressive bowing of the tibia was noted over the 4 months after removal and the rod was replaced. The area appeared fully healed 4 months after reinsertion, and the rod is being left in place for an extended time. Incidentally, she had a history of a refracture after osteotomy on her opposite extremity and was referred for bone density and calcium studies.

One patient had refracture of the osteotomy 3 weeks after rod removal after sustaining direct trauma to the leg and healed with cast application and use of an external bone stimulator. The rod had been in place for 8 months, and the bone was felt to be completely healed on preremoval radiographs.

One patient had a focal abscess developed at the site of the proximal interlocking screw, which required irrigation and debridement 2 years after the rod and screws had been removed. This subsequently healed without difficulty.

Minor complications occurred in 5/59 patients (8.5\%). All five patients had pain at a locking screw leading to early removal of the screw; four distal screws and one proximal screw were removed at an average of 5 months after the index procedure (range 4-6 months).

Fourteen of 59 patients $(23.7 \%)$ had residual symptoms related to the extremity. Three patients reported normal activities but residual discomfort in the knee and occasional NSAID use. One patient with bilateral procedures had enough residual knee pain to preclude sports participation. Two patients complained of patellar crepitance. Two patients who had their rods removed had patellar tendonitis or discomfort with kneeling.
Although none of the eight extremities with a history of prior patellar dislocation had a recurrent dislocation, one patient with a history of multiple prior bilateral dislocations reported subluxation events in the patella on one side at 21month follow-up. Another patient with no history of prior dislocation had bilateral osteotomies and despite full activities, had a "sense of subluxation" in the bilateral patellae.

Four patients complained of muscle herniation at the anterior fasciotomy. One of these had repair at the time of rod removal and the herniation was recurrent. For three of the four patients, the muscle herniation gives them an aching pain with activities.

\section{Discussion}

Treatment of symptomatic anterior knee pain in the adolescent population is characteristically conservative. Patients with excessive lateral torsion of the tibia may fail conservative management since it does not address the underlying mechanical forces at the knee caused by the torsion. Our population of patients who failed conservative measures and went onto have correction was overwhelmingly women (90\%). The excessive lateral twist in the tibia results in excessive valgus force at the knee $[5,10]$, predisposes the knee to patellar instability $[15,16]$, osteochondral defects [2] and anterior knee pain $[11,16]$. Lateral release of the patella or transfer of the tibial tubercle do not change the underlying rotation, and this may be the reason that $22 \%$ of the patients in this series continued to have symptoms despite their original procedures at other facilities.

Intramedullary rod fixation offers several possible advantages when applied to tibial rotation osteotomies. These include no cast immobilization, less soft tissue disruption at the osteotomy and early weightbearing. The complication rate in our study was $16.9 \%$ with half $(8.5 \%)$ being major complications. This compares favorably with published complication rates of tibial osteotomy of 0-43\% [1, 4-7, 9, 12-14], Table 1 . The potential complication of compartment syndrome of the leg may have been avoided by prophylactic fasciotomy of the anterior compartment but was not masked by the use of an epidural catheter postoperatively in $89.8 \%$ of the patients. However, $4 / 57$ $(7 \%)$ of those who had the fasciotomy later complained of aching at the bulge of the muscle from the fasciotomy.

Since the start of this series, all rotational osteotomies have been performed with a rod which allows axial compression at the osteotomy site. We believe that this additional stability aids healing of the diaphyseal osteotomy and improves patient comfort but do not have the numbers to make this statistical distinction. Fibular osteotomy was 
Table 1 Tibial osteotomy complications

\begin{tabular}{|c|c|c|c|c|c|c|}
\hline Study & Population & Fixation & $\begin{array}{l}\text { Number of tibial } \\
\text { osteotomies }\end{array}$ & $\begin{array}{l}\text { Complication } \\
\text { rate }\end{array}$ & $\begin{array}{l}\text { Major } \\
\text { complications }\end{array}$ & $\begin{array}{l}\text { Minor } \\
\text { complications }\end{array}$ \\
\hline This study & Tibial torsion & IM rod & 59 & $16.9 \%(10)$ & $8.5 \%(5)$ & $8.5 \%(5)$ \\
\hline Bennett et al. [1] & Tibial torsion & Cast & 32 & & $10 \%(3)$ & $38 \%(12)$ \\
\hline Delgado et al. [4] & Pan genu torsion & Staple/wire/ExFix & 13 & 0 & 0 & 0 \\
\hline Dias et al. [5] & Tibial torsion/Spina bifida & Mixed & 59 & $11.9 \%(7)$ & $5 \%(3)$ & $6.8 \%(4)$ \\
\hline Dodgin et al. [6] & Tibial torsion & Wires & 63 & $4.8 \%(3)$ & $3.2 \%(2)$ & $1.6 \%(1)$ \\
\hline Ferri-de-Barros et al. [7] & Pan genu torsion/CP & IM rod & 14 & $43 \%(6)$ & $7 \%(1)$ & $36 \%(5)$ \\
\hline Inan et al. [9] & $\mathrm{CP} /$ tibial torsion & Cast & 247 & $6.5 \%(16)$ & $1 \%(2)$ & $5.7 \%(14)$ \\
\hline Rattey and Hyndman [12] & Tibial torsion & Cast & 45 & & $11 \%(5)$ & $22 \%(10)$ \\
\hline Ryan et al. [13] & CP/Tibial Torsion & Wires/plates & 72 & $11 \%(8)$ & $1 \%(1)$ & $9.7 \%(7)$ \\
\hline Selber et al. [14] & $\mathrm{CP} /$ spina bifida & Plate & 57 & $12.2 \%(7)$ & $5.3 \%(3)$ & $7 \%(4)$ \\
\hline Server et al. [15] & Tibial torsion & Staples & 35 & $9 \%(3)$ & $5.7 \%(2)$ & $2.9 \%(1)$ \\
\hline
\end{tabular}

not necessary to make the rotational correction, which averaged $28.8^{\circ}$ (range $20^{\circ}-50^{\circ}$ ), and the intact fibula did not prevent tibial healing.

Although $23.7 \%$ of patients in last follow-up continued to have some symptoms in the knee or leg, most of these were considered to be minor and did not impact their activity level. The retrospective nature of our study does not allow us to quantitatively score the knee pain preoperatively and compare to the postoperative level, but it is our sense from patient reports that those who remain symptomatic are at a significantly lower pain level postoperatively.

In our study, we are unable to attribute the symptom improvement in patients to the osteotomy alone as $83.1 \%$ of patients had contemporaneous lateral patellar release. We believe that the osteotomy corrects the underlying mechanical aberration and that the lateral release serves to decompress the patella laterally — a tension developed from the torsional malalignment.

We believe that intramedullary rod fixation with diaphyseal osteotomy is a safe alternative to traditional methods of fixation for elective tibial rotational osteotomy.

\section{References}

1. Bennett JT, Bunnell WP, MacEwen GD (1985) Rotational osteotomy of the distal tibia and fibula. J Pediatr Orthop 5(3):294298

2. Bramer JA et al (2004) Increased external tibial torsion and osteochondritis dissecans of the knee. Clin Orthop Relat Res (422):175-179
3. Bruce WD, Stevens PM (2004) Surgical correction of miserable malalignment syndrome. J Pediatr Orthop 24(4):392-396

4. Delgado ED et al (1996) Treatment of severe Torsional malalignment syndrome. J Pediatr Orthop 16(4):484-488

5. Dias L et al (1984) Rotational deformities of the lower limb in myelomeningocele. Evaluation and treatment. J Bone Joint Surg Am 66(2):215-223

6. Dodgin DA et al (1998) Distal tibial/fibular derotation osteotomy for correction of tibial torsion:review of technique, results in 63 cases. J Pediatr Orthop 18(1):95-101

7. Ferri-de-Barros F, Inan M, Miller F (2006) Intramedullary nail fixation of femoral and tibial percutaneous rotational osteotomy in skeletally immature adolescents with cerebral palsy. J Pediatr Orthop 26(1):115-118

8. Gordon JE et al (2005) Diaphyseal derotational osteotomy with intramedullary fixation for correction of excessive femoral anteversion in children. J Pediatr Orthop 25(4):548-553

9. Inan $\mathrm{M}$ et al (2005) Correction of rotational deformity of the tibia in cerebral palsy by percutaneous supramalleolar osteotomy. J Bone Joint Surg Br 87(10):1411-1415

10. Lim R et al (1998) Valgus knee stress in lumbosacral myelomeningocele:a gait-analysis evaluation. J Pediatr Orthop 18(4):428-433

11. Meister K, James SL (1995) Proximal tibial derotation osteotomy for anterior knee pain in the miserably malaligned extremity. Am J Orthop 24(2):149-155

12. Rattey T, Hyndman J (1994) Rotational osteotomies of the leg: tibia alone versus both tibia and fibula. J Pediatr Orthop 14(5):615-618

13. Ryan DD et al (2005) Results of tibial rotational osteotomy without concomitant fibular osteotomy in children with cerebral palsy. J Pediatr Orthop 25(1):84-88

14. Selber P et al (2004) Supramalleolar derotation osteotomy of the tibia, with $\mathrm{T}$ plate fixation. Technique and results in patients with neuromuscular disease. J Bone Joint Surg Br 86(8):1170-1175

15. Server $F$ et al (1996) Medial rotational tibial osteotomy for patellar instability secondary to lateral tibial torsion. Int Orthop 20(3): $153-158$

16. Turner MS, Smillie IS (1981) The effect of tibial torsion of the pathology of the knee. J Bone Joint Surg Br 63-B(3):396-398 